\title{
HeberFERON, a promising choice for the treatment of basal cell carcinoma
}

\author{
Iraldo Bello Rivero* \\ Center for Genetic Engineering and Biotechnology, Havana, Cuba
}

Since the discovery of interferons (IFNs) numerous laboratory investigations indicated that these proteins produced by the human organism as part of immune defense mechanism against danger signals (viral or bacterial infections, tumor growth, tissues damage and others), had great potential as antitumor agents.

Its use to fight almost all types of cancer became very common among doctors who treated these patients. The first years of studies gave clear evidence of the anti-tumor potential of IFNs; however the expected impact on the cure of cancer did not materialize. Only a few types of tumors showed significant antitumor response, as were chronic myeloid leukemia, melanoma and renal cell carcinoma.

Cuba was inserted in that battle since the 80 s when it was possible to produce natural IFN alpha for the first time from human leukocytes. Investigations were then initiated for the production of other IFNs, from leukocytes and via recombinant DNA.

The work with the IFNs in the treatment of cancer was intense in the world, but the lack of results in the cure of this or the prolongation of the survival of the patients was extinguishing the flame that gave light to these investigations. It was necessary a change in the use of these powerful molecules to assert their intrinsic antitumor power, identified since their discovery.

The HeberFERON arised as a need to obtain more effective anti-tumor products based on IFNs. The use IFNs had not achieved the expected results in the treatment of cancer. The combination of several types of IFNs, empirically, also failed to properly exploit the potentiation of their effects.

In this context, at the Center for Genetic Engineering and Biotechnology (CIGB), a group of enthusiastic and committed researchers started studying how to optimize the combined use of the recombinant IFNs alfa2b and gamma, produced at the CIGB.

In 2005, these efforts were crowned with the presentation of a patent describing the obtaining of a new formulation of IFNs, based on the synergism of the combination of the IFNs alpha $2 \mathrm{~b}$ and gamma and in an ingenious work of identification of excipients, which allowed to obtain a new formulation of IFNs, unique in the world, with a stronger antitumor action than separately IFNs and more safely, during the treatment of patients [1].

From that moment on, a rapid career that lasted 13 years, it was possible to have a group of data in the field of cellular and molecular biology and clinical research, which distinguishes HeberFERON as a promising choice for the treatment of cancer.

HeberFERON consists of the combination, in synergistic (potentiating) proportions of anti-proliferative (anti-tumor) activity, of recombinant IFNs alpha $2 \mathrm{~b}$ and gamma. The IFNs are co-lyophilized in a single bottle.

The HeberFERON eliminates or reduces the tumor mass. The antitumor response is achieved faster, and is longer, than that of observed for IFNs separately, as an expression of its higher biological potency. Its use in the therapy of BCCs in the periocular area avoids complex surgeries and their sequelae, with a good safety profile. At the same time it leaves an excellent aesthetic in the injection site. These characteristics offer patients a better quality of life. The HeberFERON also exercises these effects remotely. Injuries near or in the environment are reduced or cured

The HeberFERON should be administered 3 times per week for 3 weeks, peritumorally, intradermally. The final evaluation must be done in week 16 after the start of treatment.

The main adverse events that can be attributed to HeberFERON with a frequency greater than $10 \%$ are fever $(64.4 \%)$, chills $(49.7 \%)$, arthralgia (35.6\%), headache (32.2\%), asthenia (29.5\%), general malaise $(24.8 \%)$, anorexia $(21.5 \%)$, perilesional edema and erythema $(17.4 \%)$, myalgia (14.1\%), nausea (12.1\%) and diarrhea (10.7\%).

\section{Summary of clinical development}

The first clinical study (InCarbacel-II, 2001) of the combination of recombinant alpha2b and gamma IFNs (CIGB-128) was performed in 60 patients with surgical $\mathrm{BCC},<4 \mathrm{~cm}$. It was a randomized, controlled and double-blind study, where patients were treated for 3 weeks with a dose of 1.75 MUI. In this study, IFN alpha2b, IFN gamma and CIGB128 were compared. The combination produced a greater number (42\%) of complete responses with respect to IFN alpha2b (33\%). The first CRs were observed 1 month earlier in the CIGB-128 group [2].

A second study (InCCNM-I) was conducted in 2002. This was a case treatment study, in 16 patients with advanced BCC or squamous cell carcinoma (SCC), with lesions between $2-21 \mathrm{~cm}$ in the longest dimension. In this study, a $\mathrm{CR}=47 \%$ was obtained; and a partial response $(\mathrm{PR})=40 \%$. The duration of the response in these patients was more than 38 months on average [3].

In 2009, the first Pharmacokinetics and Pharmacodynamics (FC/ FD) study was carried out with HeberFERON. This was carried out within the framework of the MYFIC study, in patients with a clinical

${ }^{*}$ Correspondence to: Iraldo Bello Rivero, Center for Genetic Engineering and Biotechnology, Havana, Cuba, E-mail: Iraldo.bello@cigb.edu.cu

Received: October 16, 2018; Accepted: October 26, 2018; Published: October 30,2018 
and histological diagnosis of Mycosis Fungoides in stages IB, IIA, IIB, and III.

For the study of FC/FD, a single initial dose of 23 MUI was administered intramuscularly. After the FC the patients continued the treatment for their disease with a treatment divided into an induction stage and a maintenance one with 11.5 MUI of HeberFERON.

The PK/PD study demonstrated for the first time in the world that the FC of the alpha2b and gamma IFNs administered as a whole did not interfere and that the FD is potentiated 5-10 times compared to the IFNs separately. This result confirmed in vivo the biological potency of this new formulation of IFNs [4]. Another PK/PD study in healthy volunteers with HeberFERON confirmed the precedent data [5].

In the case of the clinical treatment of patients with mycosis fungoides for 12 months intramuscularly with HeberFERON, only one patient progressed (8.3\%) and the overall clinical response was $83.3 \%$ $(10 / 12)$. The average time to the overall response was 13 weeks. Quality of life was good in $90 \%$ of patients.

In 2010, the dose study, InCarbacel-III, was carried out, with the inclusion of 75 patients with lesions between 1.0 and $5 \mathrm{~cm}$. There were $60 \%$ and $85 \%$ of CRs confirmed by histology, in the highest dose groups (7.0 and 10.5 MUI, respectively) [1]. During follow-up until 4 years, only $0.03 \%$ had recurrence of the lesion with a complete response. A decrease in the appearance of new lesions has also been observed in 5 times compared to that described for this type of patients, where $40 \%$ of these develop a second BCC.

Also in 2010, the CECIN study was started in 27 patients with SCC stages I and II. In this treatment with 11.5 MUI of HeberFERON 3 times per week for 3 weeks, $51.9 \%$ was obtained and objective response $(\mathrm{ORR}=\mathrm{CR}+\mathrm{PR})$ of $66.7 \%$. At follow-up at 4 years, no patient had recurrence of the cured lesion.

HeberFERON was registered in Cuba in 2016 for the treatment of basal cell carcinomas (BCC) of any subtype, size and location, as well as combined with other procedures, surgical or not (chemotherapy and/ or radiotherapy). So far, more than 1354 patients with BCC have been treated HeberFERON, of them 161 were advanced BCC, 681 high risk BCC and 181 patients with multiple (more than 5 lesions) BCC, in the $50 \%$ of cases mutilations in ear, nose or eyelid were avoided.

\section{References}

1. Patent application. Stable formula- tions containing enhancing proportios of gamma and alpha interferons. WO 20 07/051431 (10.05.20 07 Gazette 2007/19).

2. Bello-Rivero I, Garcia-Vega Y, Valenzuela-Silva C, Bello-Alvarez C, VázquezBlomquist D, et al. (2013) Development of a new formulation of interferons (HEBERPAG) for BCC treatment. Cancer Res Ther 10: 235-243.

3. Anasagasti-Angulo L, Garcia-Vega Y, Barcelona-Perez S, Lopez-Saura P, BelloRivero I (2009) Treatment of advanced, recurrent, resistant to previous treatments basal and squamous cell skin carcinomas with a synergistic formulation of interferons. Open, prospec- tive study. BMC Cancer 9: 262. [Crossref]

4. García-Vega Y, García-García I, Collazo-Caballero SE, Santely-Pravia EE, CruzRamírez A, et al. (2012) Pharmacokinetic and pharmacodynamic characterization of a new formulation containing syn- ergistic proportions of interferons alpha- $2 b$ and gamma (HeberPAG) in pa- tients with mycosis fungoides: an open-label trial. $B M C$ Pharmacol Toxicol 13: 20. [Crossref]

5. García-García I, Hernández-González I, Díaz-Machado A, González-Delgado CA, Pérez-Rodríguez S, et al. (2016) Pharmacokinetic and pharmacodynamic characterization of a novel formulation containing co-formulated interferons alpha- $2 \mathrm{~b}$ and gamma in healthy male volunteers. BMC Pharmacol Toxicol 17: 58. [Crossref]

Copyright: (C2018 Rivero IB. This is an open-access article distributed under the terms of the Creative Commons Attribution License, which permits unrestricted use, distribution, and reproduction in any medium, provided the original author and source are credited. 